\title{
Test, Track and Treat the Devil in the Paranasal Sinuses
}

https://doi.org/10.47210/bjohns.2021.v29i1.3

Anilkumar Harugop, ${ }^{1}$ Rajesh Radhakrishna Havaldar, ${ }^{1}$ O.Padmavathy, ${ }^{1}$ Reshma Ramanan ${ }^{1}$

\begin{abstract}
Introduction
Complications of rhinosinusitis result from progression of acute fungal or bacterial rhinosinusitis beyond the paranasal sinuses, potentially causing significant morbidity from either local or distant spread.

Case Report

A $45 y$ year old male patient presented with left sided headache since 8 days, left sided facial pain and swelling since 4 days which rapidly progressed to have pre-maxillay pus pointing and cellulitis. Functional endoscopic sinus surgery (FESS) was planned and intraoperatively inferior turbinectomy and maxillary clearance through the necrosed inferior turbinate was performed. Discussion

The management of acute fungal rhinosinusitis is surgical debridement by doing FESS. This case report highlights that it may not be the case always. The treatment in the form of maxillary clearance and debridement of necrosed area need to be tailor made according to the disease and involvement as suggested on radiological imaging.

$\underline{\text { Keywords }}$

Sinusitis; Mucormycosis; Subcutaneous Cellulitis
\end{abstract}

ABSTRACT

$\mathrm{C}$ omplications of rhinosinusitis result from progression of acute fungal or bacterial rhinosinusitis beyond the paranasal sinuses, potentially causing significant morbidity from either local or distant spread. ${ }^{1}$ An infection from sinuses can easily spread to the orbit and to the intracranial cavity as these anatomical structures are very closely interrelated. If it occurs in elderly individuals the cause may be due to uncontrolled diabetes, immunocompromised status, fungal infections, untreated long-standing disease, late presentation and inappropriate medical line of management. Acute invasive fungal rhinosinusitis (AIFR) is a potentially fatal infection that affects immunocompromised patients. If AIFR is not treated promptly it can lead to serious complications. One of the

1 - Department of ENT, J.N.Medical College, KLE Academy of Higher Education \& Research, Belagavi, Karnataka

\section{Corresponding author:}

Dr O.Padmavathy

email: paduop@gmail.com most important complications include orbital cellulitis. The commonest clinical manifestation would be proptosis with or without restricted eyeball movements, in some cases proptosis may not be evident and computed tomography (CT) orbit will help to know the extent and involvement of orbital contents. ${ }^{2}$

\section{Case Report}

A 45 year male patient presented with left sided headache since 8 days, left sided facial pain and swelling since 4 days. The facial swelling was diffuse over the left cheek region which rapidly progressed to involve the left eye.

Patient was not a diabetic or hypertensive. His random blood sugar was $395 \mathrm{mg} / \mathrm{dl}$ and a physician referral was given for the same. Patient was conscious and oriented.

On examination there was a diffuse swelling over the left middle third of the face which extended mediolaterally from the lateral aspect of the nose to the lateral canthus of the eye and superoinferiorly from the infraorbital region to $1 \mathrm{~cm}$ above the left corner of the 


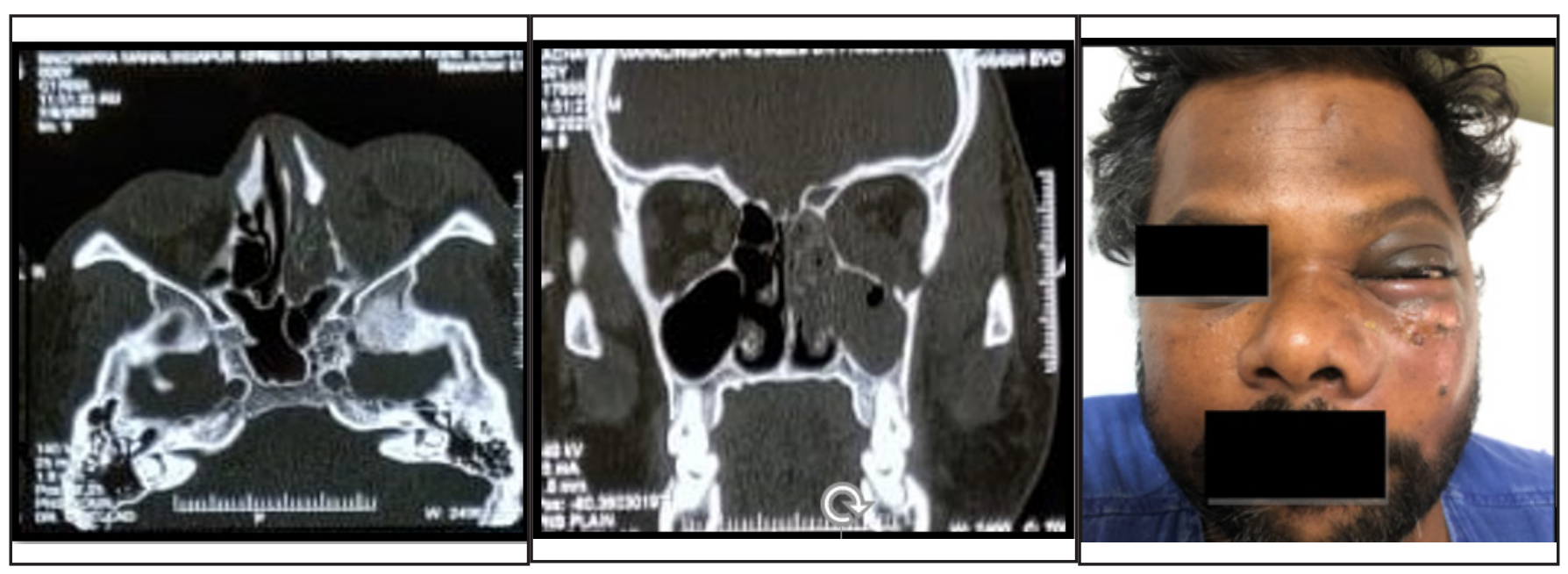

Fig. 1. CT PNS axial view showing collection in the left ethmoids with periorbital edema
Fig. 2. CT PNS coronal view showing involvement of left inferior turbinate, middle meatus and maxillary sinus with no involvement of orbital floor and lamina papyracea
Fig. 3. Image showing swelling with pus pointing in left infraorbital region mouth. The skin over the swelling was erythematous and tense. On palpation the swelling was tender with local rise of temperature. Left sided periorbital oedema was also noted and eye opening was reduced. However, eye movements were normal and pupils were reactive. Intraoral examination did not reveal any abnormalities apart from tobacco stained tooth. Intranasal examination showed enlarged inferior and middle turbinate with bulge in the lateral wall of the nose touching the septum on the left side. The inferior turbinate was insensitive to touch. No purulent nasal discharge was seen. Mucosa was congested. Right nasal cavity was normal and right sided ocular movements were normal.

A CT scan of paranasal sinus (PNS) showed features of left sided frontal, maxillary and ethmoidal sinusitis. (Figs. 1 and 2)

Lab investigations revealed elevated sugars of $390 \mathrm{mg} / \mathrm{dl}$, urine ketone bodies $3+$ and $\mathrm{HbA} 1 \mathrm{c}$ of 16.8 $\%$ was noted. All other blood parameters were within normal limit. The patient was tested for COVID 19 and was found to be negative. He was started on broad spectrum antibiotics. Blood sugars were managed as per physician opinion. Daily inspection showed a progressive increase in the size of the facial swelling and increasing periorbital oedema.
On the 4th day of admission, a pus point was noted in the left infraorbital region with the overlying skin being tense. (Fig. 3) Patient was taken up for Functional Endoscopic Sinus Surgery (FESS) under general anaesthesia.

Intraoperatively, the whole of the inferior turbinate was medialised due to the extensive collection in the maxillary sinus and pus pointing was noted. (Fig 4) On giving a nick, thick mucopurulent pus was suctioned and whole of the inferior turbinate was found to be necrosed. On further clearing the pus, the maxillary sinus was opened. The medial and antero-lateral wall of the maxillary sinus was necrosed and pus filled curdy white debris were removed. (Fig. 5) Complete debridement was done along with left inferior turbinectomy and whole of the maxillary sinus was cleared. Tissue was sent for Potassium hydroxide $(\mathrm{KOH})$ staining, fungal culture and histopathological examination. Lamina papyracea was not breached.

On the first post-operative day the facial swelling had reduced, and eye opening had improved. $\mathrm{KOH}$ report was positive and patient was started on intravenous Amphotericin B at a dosage of $0.25 \mathrm{mg} / \mathrm{kg} / \mathrm{day}$ for 14 days with regular renal function test and electrolyte testing on alternate days and fluconazole nasal wash as 


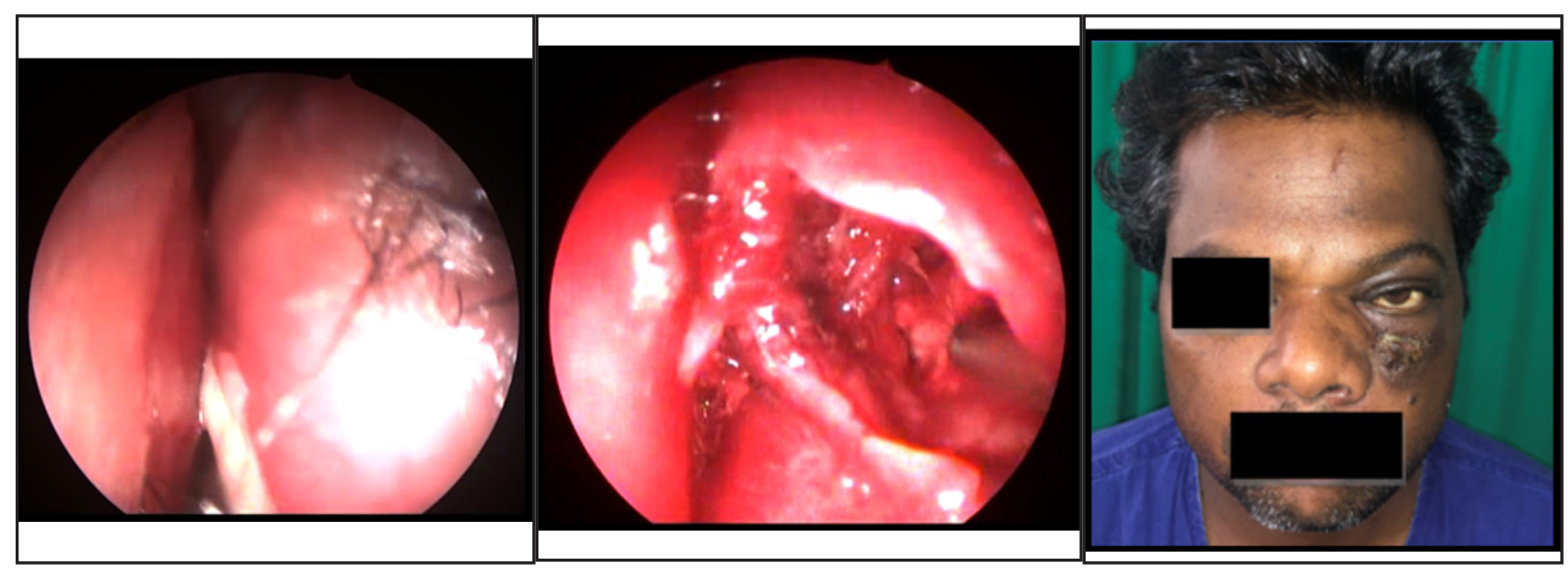

Fig. 4. DNE image of left nasal cavity showing pus filled inferior turbinate which is pushed medially touching the septum.
Fig. 5. DNE image of left nasal cavity showing complete necrosed inferior turbinate and medial wall of maxillary sinus.
Fig. 6. Post-operative image showing complete reduction of the infra orbital swelling per our institutional protocol. The facial swelling had resolved almost completely in the next 48 hours and the periorbital oedema was minimal. Eye opening had progressively improved. (Fig. 6) The organism isolated was Mucor species.

\section{Discussion}

The complications of acute invasive fungal rhinosinusitis are potentially life threatening. Complications are generally classified as orbital, intra-cranial, bony or chronic. Complications may be caused by either local progression or distant spread via the bloodstream. Local progression is typically through areas where the surrounding bone is thin such as the porous lamina papyracea, where there is a direct anatomical connection or through osteitic bone. ${ }^{1,2}$

The most common complication of sinusitis is orbital cellulitis followed by intracranial complications like meningitis, brain abscess and cavernous sinus thrombosis. If intra-orbital complications are not treated in time, they can progress to life-threatening complications such as optic neuritis, cavernous sinus thrombophlebitis or intracranial complications. A significant proportion of patients $(5-40 \%)$ can be affected by sinusitis, mostly due to the delayed diagnosis of the disease. ${ }^{3,4}$

Acute invasive fungal rhinosinusitis (AIFR) is a potentially fatal infection that affects immunocompromised patients. Multiple fungal species have been identified in patients with AIFR among which Aspergillus and Mucoraceae are the most common pathogens.., 6

In a 15 year review of 90 patients with orbital cellulitis complicating sinusitis done by Nwaorgu (2004), sinuses were the origin of the infection in 57\% patients. ${ }^{7}$

In a study of 218 patients of orbital cellulitis, done by Choudhary et al. (2007), it was found that sinusitis was the most common predisposing factor. ${ }^{8}$

Based upon the anatomical sites and the degree of involvement, the patient can have various presentations in complicated sinusitis. Along with a proper history and clinical examination, extensive radiological evaluation CT scan/magnetic resonance imaging (MRI) of the paranasal sinus and brain is always mandated in patients with suspected complications. Conservative treatment is offered as the first line of treatment for all complicated sinusitis. Endoscopic/open surgical drainage is warranted in progressive disease which does not respond to medical treatment. ${ }^{9,10}$ 
In our case, upon seeing a pus pointing area from a necrosed inferior turbinate, with a detailed pre-operative imaging and knowledge of anatomical structures in the close vicinity, an attempt was made to clear the maxillary sinus directly and later proceed with other conventional steps of FESS as required. The benefits were an immediate reduction in the volume occupied by the disease which hampered visualisation of the nasal cavity and the lateral nasal wall. Upon clearing the contents from the maxilla, the visualisation of the lamina papyracea was more clear and a wise decision was made to not proceed with orbital decompression as it was not affected.

If appropriate treatment is not done, acute invasive fungal sinusitis can lead to intra orbital and intracranial complications such as orbital cellulitis, orbital apex syndrome, cavernous sinus thrombosis etc. If untreated, $50-80 \%$ mortality rates from intra orbital and intra cranial complications are reported. ${ }^{9}$

\section{Conclusion}

Although the conventional protocol of managing these cases is by doing functional endoscopic sinus surgery (FESS) and proceeding with orbital decompression if the orbits are found to be involved on radiological imaging or during endoscopic visualisation, this report highlights that it may not be the case always. Attempt should be made to clear the disease directly and later proceed with other conventional steps of FESS as required thereby preventing the damage to vital structures. Hence, we suggest a tailor-made concept of clearing the disease in the nasal cavity and the para nasal sinuses in order to achieve the prime objective of disease clearance and also aid in better visualisation of the more posterior and superior structures in the lateral nasal wall thus preventing unnecessary iatrogenic potentially lethal complications.

\section{References}

1. Pradhan P, Samal DK, Preetam C, Parida PK. Intraorbital and Intracranial Complications of Acute Rhinosinusitis: A Rare Case Report. Iran J Otorhinolaryngol. 2018;30(100):301-4

2. Harugop AS, Bellad SA, Sethunath D, Havaldar RR, Reddy D. Sinusitis with Extensive Neck, Orbit \& Scalp Involvement - A Rare Presentation. Madridge J Otorhinolaryngol. 2018; 4(1): 70-72

3. Younis RT, Lazar RH, Bustillo A, Anand VK. Orbital infection as a complication of sinusitis: are diagnostic and treatment trends changing. Ear Nose Throat J. 2002; 8(11): 771-5

4. Harugop AKS, Mudhol RS, Bhimanapati D, Soni S, Bellad SA Achuta A, et al. Varied orbital manifestations of paranasal sinus disease. Int J Otorhinolaryngol Head Neck Surg. 2019; 5:10614

5. Süslü AE, Oğretmenoğlu O, Süslü N, Yücel OT, Onerci TM Acute invasive fungal rhinosinusitis: our experience with 19 patients. Eur Arch Otorhinolaryngol. 2009; 266(1):77-82

6. Arora V, Nagarkar NM, Dass A, Malhotra A. Invasive rhinoorbital aspergillosis. Indian Journal of Otolaryngology and Head \& Neck Surgery 2011; 63(4):325-9

7. Nwaorgu OGB, Awobem FJ, Onakoya PA, Wabem AAA Orbital cellulitis complicating sinusitis: a 15 year review. Niger J Surg Res. 2004; 6(1-2): 14-6

8. Chaudhry IA, Shamsi FA, Elzaridi E, et al. Outcome of treated orbital cellulitis in a tertiary eye care centre in the middle East. Ophthalmology 2007; 114(2): 345-54

9. Stuart AJ, Lloyd GW. Mucormycosis presenting as orbital cellulitis; a case report. Adv Ophthalmol Vis Syst. 2015;3(1):249-51

10. Wan Y, Shi G, Wang H. Treatment of orbital complications following acute rhinosinusitis in children. Balkan Med J. 2016; 33: 401-6. 